\title{
Call for worldwide withdrawal of tiotropium Respimat mist inhaler
}

In the print version of this Editorial we mistakenly used a picture of the HandiHaler (rather than the Respimat) device, which we accompanied with an incorrect caption (BMJ 2012;345:e7390, doi:10.1136/bmj.e7390). The caption "Use of the Respimat device confers greatest risk" is inaccurately applied to the picture of the HandiHaler device. We would like to clarify that the article refers to the risks of the Respimat device and that the authors do not question the use of the HandiHaler. We apologise for any confusion caused by this mistake.

Cite this as: BMJ 2012;345:e8073

๑ BMJ Publishing Group Ltd 2012 